\title{
Effects of Te- and Fe-doping on the superconducting properties in FeySe1-xTex thin films
}

\section{Yalin Zhang}

Nanjing University

\section{Tong Wang}

Nanjing University of Aeronautics and Astronautics

Zhihe Wang ( $\nabla$ zhwang@nju.edu.cn )

Nanjing University

Zhongwen Xing

Nanjing University

\section{Research Article}

Keywords: feyse1, xtex, films, composition, thin, chemical

Posted Date: August 30th, 2021

DOI: https://doi.org/10.21203/rs.3.rs-847481/v1

License: (c) (i) This work is licensed under a Creative Commons Attribution 4.0 International License. Read Full License 


\title{
Effects of Te- and Fe-doping on the superconducting properties in $\mathrm{Fe}_{y} \mathrm{Se}_{1-x} \mathrm{Te}_{x}$ thin films
}

Yalin Zhang ${ }^{1,2}$, Tong Wang ${ }^{3}$, Zhihe Wang ${ }^{1,4^{*}}$, and Zhongwen Xing ${ }^{1,5^{*}}$

${ }^{1}$ National Laboratory of Solid State Microstructures and Collaborative Innovation Center of Advanced Microstructures, Nanjing University, Nanjing 210093, China

${ }^{2}$ College of Engineering and Applied Sciences, Nanjing University, Nanjing 210093, China ${ }^{3}$ Department of Mathematics, Nanjing University of Aeronautics and Astronautics, Nanjing 210016, China ${ }^{4}$ School of Physics, Nanjing University, Nanjing 210093, China

${ }^{5}$ School of Electronic Science and Engineering, Nanjing University, Nanjing 210093, China

\begin{abstract}
High quality $\mathrm{Fe}_{y} \mathrm{Se}_{1-x} \mathrm{Te}_{x}$ epitaxial thin films have been fabricated on $\mathrm{TiO}_{2}$-buffered $\mathrm{SrTiO}_{3}$ substrates by pulsed laser deposition technology. There is a significant composition deviation between the nominal target and the thin film. Te doping can affect the Se/Te ratio and Fe content in chemical composition. The superconducting transition temperature $T_{c}$ is closely related to the chemical composition. Fe vacancies are beneficial for the $\mathrm{Fe}_{y} \mathrm{Se}_{1-x} \mathrm{Te}_{x}$ films to exhibit the higher $T_{c}$. A 3D phase diagram is given that the optimize range is $x=0.13-0.15$ and $y=0.73-0.78$ for $\mathrm{Fe}_{y} \mathrm{Se}_{1-x} \mathrm{Te}_{x}$ films. The anisotropic, effective pining energy and critical current density for the $\mathrm{Fe}_{0.72} \mathrm{Se}_{0.94} \mathrm{Te}_{0.06}, \mathrm{Fe}_{0.76} \mathrm{Se}_{0.87} \mathrm{Te}_{0.13}$ and $\mathrm{Fe}_{0.91} \mathrm{Se}_{0.77} \mathrm{Te}_{0.23}$ samples were studied in detail. The scanning transmission electron microscopy images display a regular pattern without obviously scale defects at the interfacial structure.
\end{abstract}


In 2008, Hosono et al. [1] first discovered the iron-based superconductor $\mathrm{LaO}_{1}$ ${ }_{x} \mathrm{~F}_{x} \mathrm{FeAs}$, which has a superconducting critical temperature of $26 \mathrm{~K}$. Subsequently, Hsu et al. [2] reported that the binary superconductor FeSe with antifluorite planes and has the transition temperature of $8 \mathrm{~K}$. Through the applied pressure on the samples, the transition temperature can reach $\sim 37 \mathrm{~K}[3,4]$. Xue et al. [5] reported a superconducting transition temperature above $100 \mathrm{~K}$ in single-layer FeSe film grown on a doped $\mathrm{SrTiO}_{3}$ (STO) substrate by molecular beam epitaxy method. Due to its simple crystal structure, this binary FeSe system with higher $T_{c}$ are available has attracted tremendous interest in exploring the mechanism of high-temperature superconductivity [6-8]. Generally, the FeSe layer is responsible for the superconductivity and the paired electrons are mainly $3 \mathrm{~d}$ electrons of Fe ions. Meanwhile, the FeSe layers exhibit electrical neutrality and the atoms between the layers are bonded together by van der Waals [9, 10]. However, the same structure as FeTe does not show superconducting behavior. Yeh et al. [11] found that the Te atoms are replaced by partially substituted Se atoms, the antiferromagnetic can be suppressed and superconductivity is induced with a superconducting transition temperature of $15 \mathrm{~K}$. In bulk crystals, the optimal Te content to achieve the highest $T_{c}$ is considered to be $x \approx 0.6$, and phase separation occurs in the region of $0.1 \leq x \leq 0.3$ [12]. Liu et al. [13] have studied the electronic and magnetic phase diagram of $\mathrm{Fe}_{1.02} \mathrm{Se}_{x} \mathrm{Te}_{1-x}$ single crystal superconductors. They showed that the phase diagram contains three regions, namely long-range antiferromagnetic order with a wave vector $(\pi, 0)$ in region I $(0 \leq x<0.09)$, neither long-range antiferromagnetic order nor bulk superconductivity in Region II $(0.09<x<0.29)$ and the evidence of bulk superconductivity with the $T_{c}$ about $14.5 \mathrm{~K}$ in Region III $(x \geq 0.29)$. The phase diagram of $\mathrm{FeSe}_{1-x} \mathrm{Te}_{x}$ films on $\mathrm{CaF}_{2}$ substrates showed that the maximum value of $T_{c}$ is as high as $23 \mathrm{~K}$ at $x=0.2$, and a sudden suppression of $T_{c}$ is observed at $0.1<x<0.2$, whereas $T_{c}$ increases with decreasing $x$ for $0.2 \leq x<1$ [14]. The interface effect between film and substrate makes it possible to obtain the $\mathrm{Fe}_{y} \mathrm{Se}_{1-x} \mathrm{Te}_{x}$ films with high transition temperature in a metastable phase. Although researchers have reported many studies on superconducting mechanism of $\mathrm{Fe}(\mathrm{Se}, \mathrm{Te})$ films that papered by pulsed laser deposition (PLD), the bidirectional effect of chemical composition on the superconductivity of 
$\mathrm{Fe}_{y} \mathrm{Se}_{1-x} \mathrm{Te}_{x}$ films is uncertain [15-19]. In this paper, we have prepared different polycrystalline targets to grow $\mathrm{Fe}_{y} \mathrm{Se}_{1-x} \mathrm{Te}_{x}$ films and did a detailed investigation on the superconducting properties and its phase diagram. The experimental results show that there is a significant deviation between the nominal compositions of polycrystalline targets and the real compositions of films. The increase of Te doping can have an impact not only on $\mathrm{Se} / \mathrm{Te}$ ratio but also Fe content. The electrical transport results indicate that the optimal range of Te and Fe content is $x=0.13-0.15$ and $y=0.73-0.78$ for $\mathrm{Fe}_{y} \mathrm{Se}_{1}$ ${ }_{x} \mathrm{Te}_{x}$ films with excellent superconductivity. As $x=0.13, y=0.76$, the maximum of zeroresistivity temperature $T_{c}{ }^{0} \max$ of film is over $17 \mathrm{~K}$, the upper critical field $H_{c 2}(0) \approx 159.5$ $\mathrm{T}$, and the critical current density $J_{c}$ is higher than $10^{6} \mathrm{~A} / \mathrm{cm}^{2}$ at $4 \mathrm{~K}$. Moreover, the STEM images reveal that the interface region of $\mathrm{Fe}_{y} \mathrm{Se}_{1-x} \mathrm{Te}_{x} / \mathrm{TiO}_{2} / \mathrm{SrTiO}_{3}$ heterostructure is sharp and clear, and no obvious atomic diffusion and migration was detected.

\section{Results and discussion}

In the published papers [14, 20-22], authors usually defined the nominal composition of the targets as the real composition for $\mathrm{Fe}_{y} \mathrm{Se}_{1-x} \mathrm{Te}_{x}$ films. However, the deviation between the nominal composition and the real composition may affect the study on the mechanism of superconductivity for $\mathrm{Fe}_{y} \mathrm{Se}_{1-x} \mathrm{Te}_{x}$ films. We determined the real composition of films by EDX mapping in SEM technology. Our experimental results show that there is a significant deviation between the nominal composition and the real composition in the two groups, as shown in tables 1 and 2. At the first, we fixed the content of Fe and adjusted the amount of Te doping in the targets (nominal composition in table 1). EDX results show that Te doping can have an impact not only on Se/Te ratio but also the Fe content in films. The optimal chemical composition may play an important role in films with excellent superconducting property. Base on this result, we measured the superconducting properties of these films and gave them in the following text. To explore the effect of Fe content on the superconductivity of $\mathrm{Fe}_{y} \mathrm{Se}_{1-}$ ${ }_{x} \mathrm{Te}_{x}$ films, we fixed the $\mathrm{Se} / \mathrm{Te}$ ratio and change the $\mathrm{Fe}$ doping in the nominal composition, as shown in Table 2. It can be seen that the change of Fe doping in the nominal composition also leads to the change of the Fe content in the real composition, 
but has little influence on the ratio of Se/Te. During the deposition, the transfer and growth rate of $\mathrm{Fe} / \mathrm{Se} / \mathrm{Te}$ elements are different, which may lead to the obvious deviation of chemical composition between target and film. Therefore, we think that it is inaccurate to directly define the nominal composition of the targets as the real composition of the films.

The XRD patterns of $\mathrm{Fe}_{y} \mathrm{Se}_{1-x} \mathrm{Te}_{x}$ films are shown in Fig. 1. From Fig. 1, only $\mathrm{Fe}_{y} \mathrm{Se}_{1-x} \mathrm{Te}_{x}$ and $\mathrm{TiO}_{2}$ peaks are observed along the $c$-axis $(00 l)$, which indicates the $\mathrm{Fe}_{y} \mathrm{Se}_{1-x} \mathrm{Te}_{x}$ epitaxial films to be single tetragonal phase. Our previous work confirmed that $\mathrm{TiO}_{2}$ as a buffer layer could increase the lattice match between $\mathrm{Fe}(\mathrm{Se}, \mathrm{Te})$ film and STO substrate, so as to enhance the superconducting property of $\mathrm{Fe}(\mathrm{Se}, \mathrm{Te})$ film [23]. We find that with increasing Te doping, the $(00 l)$ peaks significantly shift to a low angle. The $c$-axis lattice parameters for $\mathrm{Fe}_{y} \mathrm{Se}_{1-x} \mathrm{Te}_{x}$ films were obtained by fitting the (001) peak, as listed in table 1. The ionic radius of $\mathrm{Te}\left(\mathrm{Te}^{2-}, 221 \mathrm{pm}\right)$ is larger than that of $\mathrm{Se}$ $\left(\mathrm{Se}^{2-}, 198 \mathrm{pm}\right)$ [24]. Te doping can increase the distance between the Fe plane and Se (Te) atom $\left(h_{\mathrm{Fe}-\mathrm{Se} / \mathrm{Te}}\right)$, which result in the increase of $c$-axis lattice parameters. Zhuang et al [25] and Ima et al [26] have reported the effect of chemical composition on the structure in $\mathrm{FeSe}_{1-x} \mathrm{Te}_{x}$ films. In our results, the increase of Te doping in targets can also raise the Fe content in the $\mathrm{Fe}_{y} \mathrm{Se}_{1-x} \mathrm{Te}_{x}$ films. For $y>1$ in table 1, we assume that the additional Fe may be incorporated in the inter-layer of Fe-Se/Te space. Thus, Fe content play a part in the change of lattice parameter. Shi. et al [22] assumed that two key factors affected the lattice parameters of thin films under the Fe-deficient conditions. The ionic radius of $\mathrm{Fe}$ is smaller than that of $\mathrm{Se}$ and Te. Fe vacancy phase leads to a smaller $c$-axis lattice parameter, while $\mathrm{Se} / \mathrm{Te}$ interstitial phase leads to a larger $c$-axis in comparison with the stoichiometric phase. For table 2, with increasing the Fe doping, the $c$-axis lattice parameter of films increases. The above results show that the superconducting structures of $\mathrm{Fe}_{y} \mathrm{Se}_{1-x} \mathrm{Te}_{x}$ films are not changed with $0.63<y<1.43$, whereas $\mathrm{Te}$ and $\mathrm{Fe}$ doping jointly influence the $c$-axis lattice parameter.

Figure 2 (a) shows the temperature dependence of the normalized resistivity $\rho / \rho_{300 \mathrm{~K}}(\rho-\mathrm{T})$ for the $\mathrm{Fe}_{y} \mathrm{Se}_{1-x} \mathrm{Te}_{x}$ films. For $0.03 \leq x \leq 0.23$ in Fig. 2 (a), as the temperature above the superconducting transition, the films only display metallic 
behavior. However, for $x>0.23$, the resistivity of films changes from semiconducting to metallic before superconducting transition. This change may attribute to the structure phase transition and magnetic phase transition that caused by Te doping. If we define the point of intersection of the two lines as the normal-state resistivity $\rho_{\mathrm{n}}$, as shown in the inset of Fig. 2 (a), the onset transition temperature $T_{c}{ }^{\text {onset }}$ and zero-resistivity temperature $T_{c}{ }^{0}$ are obtained from these $\rho$-T curves where the resistivity is $90 \%$ and $1 \%$ of the normal state resistivity $\rho_{\mathrm{n}}$, respectively. The values of $T_{c}{ }^{\text {onset }}$ and $T_{c}{ }^{0}$ for these samples were listed in table 1 and plotted in the 3D phase diagram, as shown in Fig. 2 (c). With increasing the Te doping, the $T_{c}$ rises at first and then decreases. From Fig. 2 (c), the $\mathrm{Fe}_{0.76} \mathrm{Se}_{0.87} \mathrm{Te}_{0.13}$ film exhibits the higher $T_{c}{ }^{\text {onset }}$ and $T_{c}{ }^{0}$ about $18.95 \mathrm{~K}$ and 17.34 $\mathrm{K}$, respectively. Surprising us, the composition of the $\mathrm{Fe}_{0.76} \mathrm{Se}_{0.87} \mathrm{Te}_{0.13}$ film is not consistent with that of the single crystal, where the highest $T_{c}$ is considered $x \approx 0.6$ in $\mathrm{Fe}\left(\mathrm{Se}_{1-x} \mathrm{Te}_{x}\right)_{0.82}$ polycrystal sample, and located at the phase separation region of $0.1 \leq$ $x \leq 0.3$ [12]. They argued that the single-phase of $\mathrm{Fe}\left(\mathrm{Se}_{1-x} \mathrm{Te}_{x}\right)_{0.82}$ single crystals with the region of $0.1 \leq x \leq 0.3$ were not easy to obtained. However, Imai reported that the single-phase epitaxial films of $\mathrm{FeSe}_{1-x} \mathrm{Te}_{x}$ with $0.1 \leq x \leq 0.4$ could be successfully prepared on $\mathrm{CaF}_{2}$ substrates, attributing to the strain effect between film and substrate [14]. Due to the different substrates, there is a difference in the suppression of phase separation and giant enhancement of $T_{c}$ for $\mathrm{Fe}_{y} \mathrm{Se}_{1-x} \mathrm{Te}_{x}$ films. Our experimental results display that the sudden suppression of $T_{c}$ is observed at $0.03 \leq x<0.13$, whereas $T_{c}$ increases with decreasing $x$ for $0.13 \leq x<0.56$. The superconducting property is related to the $\mathrm{Te}$ and $\mathrm{Fe}$ content in $\mathrm{Fe}_{y} \mathrm{Se}_{1-x} \mathrm{Te}_{x}$ film. Therefore, we must consider the effects of Fe vacancies on the superconductivity of $\mathrm{Fe}_{y} \mathrm{Se}_{1-x} \mathrm{Te}_{x}$ films.

Figure 2 (b) shows the temperature dependence of the normalized resistivity $\rho / \rho_{300 \mathrm{~K}}(\rho-\mathrm{T})$ near the optimal composition $\mathrm{Fe}_{y} \mathrm{Se}_{1-x} \mathrm{Te}_{x}$ films, where $x \sim 0.15$ and $y \sim$ 0.76. The results demonstrate the effects of Fe vacancies on the superconductivity of $\mathrm{Fe}_{y} \mathrm{Se}_{1-x} \mathrm{Te}_{x}$ films. The $T_{c}{ }^{\text {onset }}$ and $T_{c}{ }^{0}$ were listed in table 2 and plotted in the $3 \mathrm{D}$ phase diagram of Fig. 2 (b). Although we do not know the reason why the $T_{c}{ }^{\text {onset }}$ and $T_{c}{ }^{0}$ increase with decreasing the Fe content near $y=0.76$, the transition width broadens much more. This result further confirms that the optimal range is $x=0.13-0.15$ and $y$ 
$=0.73-0.78$ for the $\mathrm{Fe}_{y} \mathrm{Se}_{1-x} \mathrm{Te}_{x}$ films.

Figure 2 (c) is a new 3D phase diagram for the $\mathrm{Fe}_{y} \mathrm{Se}_{1-x} \mathrm{Te}_{x}$ films. The $3 \mathrm{D}$ phase diagram can be divided into three regions, which are superconductivity (SC), flux flow (FF), and normal state (NS), respectively. The 3D phase diagram demonstrates that the phase separation is absent, and that the optimal composition for the $\mathrm{Fe}_{y} \mathrm{Se}_{1-x} \mathrm{Te}_{x}$ film on STO substrate is not $x \approx 0.5$ and $y=1$ but $x \sim 0.13$ and $y \sim 0.76$. It should be noted that the dependence of $T_{c}$ on $x$ suddenly changes at the boundary defined by $0.03 \leq x<0.13$ in our experiment. Thus, not only the decrease of $T_{c}$ with $x \geq 0.13$ can be explained by the empirical law that shows the relation between $T_{c}$ and structural parameters, but also the sudden suppression of $T_{c}$ in films with $0.03 \leq x<0.13$ can be explained by the orthorhombic distortion results in a suppression of $T_{c}$. As reported by Imai Y et al. [9], the orthorhombic distortion is applicable to the behavior of films, if a large orthorhombic distortion is observed only in films with $0<x<0.1$, which is consistent with our result of $0.03 \leq x<0.13$. Chen et al. [27] and Bendele et al [28] pointed out that a few Fe vacancies are beneficial to improve the superconductivity and raise the superconducting transition temperature for $\mathrm{Fe}_{y} \mathrm{Se}_{1-x} \mathrm{Te}_{x}$ films. The inhomogeneous distribution of $\mathrm{Fe}$ vacancies can induce the Fe disorder effect in the films with the $y<$ 1. The first-principles calculation also showed that the Fe vacancies could effectively increase the number of electron carriers and change the electronic properties in the samples [22]. Therefore, in this experiment, the highest $T_{c}{ }^{\text {onset }}$ and $T_{c}{ }^{0}$ occurred near $y$ $=0.76$. When the Te and Fe content exceed the optimal composition, the $T_{c}{ }^{\text {onset }}$ and $T_{c}{ }^{0}$ of $\mathrm{Fe}_{y} \mathrm{Se}_{1-x} \mathrm{Te}_{x}$ films decrease. For example, as $x=0.56, y=1.43$, the $\rho$ does not down to $1 \% \rho_{\mathrm{n}}$, so the $\mathrm{Fe}_{1.43} \mathrm{Se}_{0.44} \mathrm{Te}_{0.56}$ sample only has the $T_{c}{ }^{\text {onset }}$ about $8.03 \mathrm{~K}$.

To understand the new phase diagram, we have measured the electrical transport and magnetization properties for the $\mathrm{Fe}_{y} \mathrm{Se}_{1-x} \mathrm{Te}_{x}$ films in magnetic field. Here, we choose some typical results in the next part. Figure 3 (a) and (b) present the temperature dependence of resistivity of $\mathrm{Fe}_{0.76} \mathrm{Se}_{0.87} \mathrm{Te}_{0.13}$ film in various magnetic fields up to $9 \mathrm{~T}$ applied perpendicular and parallel to the $c$-axis. With increasing the applied magnetic field, the resistive transition was broadened. At the same field, the width of superconducting transition $\Delta T_{c}$ for $H / / c$ is larger than that for $H / / a b$. This result 
indicates that the $\mathrm{Fe}_{y} \mathrm{Se}_{1-x} \mathrm{Te}_{x}$ films are anisotropic near $T_{c}$.

If we define the onset transition temperature $T_{c}{ }^{\text {onset }}$ as the critical temperature $T_{c}$, namely the field is the upper critical field $H_{c 2}$, we can get the temperature of the upper critical field near $T_{c}$. The $H-T$ phase diagram for $\mathrm{Fe}_{0.72} \mathrm{Se}_{0.94} \mathrm{Te}_{0.06}, \mathrm{Fe}_{0.76} \mathrm{Se}_{0.87} \mathrm{Te}_{0.13}$ and $\mathrm{Fe}_{0.91} \mathrm{Se}_{0.77} \mathrm{Te}_{0.23}$ films is shown in Fig. 4. Using the Werthamer-Helfand-Hohenberg (WHH) formula [29] $H_{c 2}=-0.69 T_{c}\left[d H_{c 2} / d T\right]_{T_{c}}$, the upper critical field $H_{c 2}(0)$ of $\mathrm{Fe}_{0.72} \mathrm{Se}_{0.94} \mathrm{Te}_{0.06}, \mathrm{Fe}_{0.76} \mathrm{Se}_{0.87} \mathrm{Te}_{0.13}$ and $\mathrm{Fe}_{0.91} \mathrm{Se}_{0.77} \mathrm{Te}_{0.23}$ films were estimated about 109.6 T, 159.5 T and 134.2 T, respectively. It implies that the values of upper critical field $H_{c 2}(0)$ also depend on the chemical composition of $\mathrm{Fe}_{y} \mathrm{Se}_{1-x} \mathrm{Te}_{x}$ films. From Fig. 4, The anisotropic factor $\gamma$ can be obtained according to the formula: $\gamma=\left[d H_{c 2}^{a b}(T) / d T\right] /\left[d H_{c 2}^{c}(T) / d T\right]$ near $T_{c}$. The $\gamma$ value of $\mathrm{Fe}_{0.72} \mathrm{Se}_{0.94} \mathrm{Te}_{0.06}$, $\mathrm{Fe}_{0.76} \mathrm{Se}_{0.87} \mathrm{Te}_{0.13}$ and $\mathrm{Fe}_{0.91} \mathrm{Se}_{0.77} \mathrm{Te}_{0.23}$ films are 2.59, 2.09, and 1.72, respectively. The increase of Te doping inhibits the anisotropic and enhances the isotropy in $\mathrm{Fe}_{y} \mathrm{Se}_{1-x} \mathrm{Te}_{x}$ films. Te doping may enhance the correlation between Fe-Se/Te layers, which is conducive to the transmission of electrons along the $c$-axis direction and result in the decrease in anisotropy.

The effective pining energy is an important parameter to enhance the capacity of carrying current for superconducting materials. According to the thermally activated flux flow (TAFF) theory, the $\ln \rho-1 / \mathrm{T}$ in the TAFF region can be described using an Arrhenius relation [30-32], $\rho=\rho_{0} \exp \left(-\frac{U_{0}}{K_{B} T}\right)$ where $U_{0}$ is the effective pinning energy. Figure 5 (a) and (b) shows the linear relationship between $\ln \rho$ and 1/T of the $\mathrm{Fe}_{0.76} \mathrm{Se}_{0.87} \mathrm{Te}_{0.13}$ film. From the absolute slope of $\ln \rho-1 / \mathrm{T}$ curves, we can obtain the effective pinning energy $U_{0}$ of $\mathrm{Fe}_{0.72} \mathrm{Se}_{0.94} \mathrm{Te}_{0.06}, \mathrm{Fe}_{0.76} \mathrm{Se}_{0.87} \mathrm{Te}_{0.13}$ and $\mathrm{Fe}_{0.91} \mathrm{Se}_{0.77} \mathrm{Te}_{0.23}$ films, respectively, as shown in Fig. 5 (c). It can be found that the $U_{0}$ value of $\mathrm{Fe}_{0.76} \mathrm{Se}_{0.87} \mathrm{Te}_{0.13}$ is larger than that of $\mathrm{Fe}_{0.72} \mathrm{Se}_{0.94} \mathrm{Te}_{0.06}$ and/or $\mathrm{Fe}_{0.91} \mathrm{Se}_{0.77} \mathrm{Te}_{0.23}$ in the same field. Furthermore, $U_{0}$ values for $H / / a b$ plane are much higher than that for $H / / c$ plane, indicating the flux pinning is anisotropic, and the magnetic field dependence of $U_{0}$ follows a power low $U_{0}(\mathrm{~K}) \sim H^{-\alpha}$. The parameter $\alpha$ of three samples is close for 
$H / / a b$. However, there is an obvious crossover that occurred at $H \approx 2 \mathrm{~T}$ for $H / / c$. The parameter $\alpha$ for $H / / c$ is close to 0.15 in the low field and 0.5 in the high field. Generally, the parameter $\alpha$ is related to the pinning mechanism of superconductors [33]. The core pinning is responsible for $\alpha=3 / 2$, while the linear pinning is responsible for $\alpha=1$. As $\alpha=1 / 2$, the pinning mechanism in superconductor is generally considered as the planar pinning [34]. It is possible that the flux pinning in $\mathrm{Fe}_{y} \mathrm{Se}_{1-x} \mathrm{Te}_{x}$ films is the collective flux pinning, including planar pinning and $3 \mathrm{D}$ volume cores pinning.

The critical current density $J_{c}$ is also an important parameter for high quality epitaxial superconducting films. To study the effect of chemical composition on the critical current density of $\mathrm{Fe}_{y} \mathrm{Se}_{1-x} \mathrm{Te}_{x}$ films, we have measured the magnetization hysteresis loops in fields parallel to the $c$-axis from 0 to \pm 9 T. Figure 6 shows the $M-H$ loops of $\mathrm{Fe}_{0.91} \mathrm{Se}_{0.77} \mathrm{Te}_{0.23}$ film at various temperatures. The $M-H$ loops show symmetric field dependence. As the field increases, the magnetization of film decreases.

The critical current density $J_{c}$ was estimated from the $M-H$ loops by the Bean critical state model [35]: $J_{c}=20 \frac{\Delta M}{a(1-a / 3 b)}$. Where $\triangle M=M(+)-M(-), M(+)$ and $M(-)$ are the magnetizations when sweeping fields up and down, respectively. $a$ and $b$ $(a<b)$ are the $\mathrm{Fe}_{y} \mathrm{Se}_{1-x} \mathrm{Te}_{x}$ film's cross-sectional dimension. The field dependence of the critical current density $J_{c}$ at various temperatures is shown in Fig. 7. At a constant temperature, the magnetic field $H$ dependence of critical current density $J_{c}$ follows a semi-exponent-law behavior: $J_{c} \propto H^{-\delta}$. Where $\delta$ is a parameter, meaning the decreasing rate of $J_{c}$ with the field $H$ increasing. We can see that the $J_{c}$ decreases with the field $H$ increasing. The calculated $J_{c}$ at $4 \mathrm{~K}$ and $0 \mathrm{~T}$ for $\mathrm{Fe}_{0.72} \mathrm{Se}_{0.94} \mathrm{Te}_{0.06}$, $\mathrm{Fe}_{0.76} \mathrm{Se}_{0.87} \mathrm{Te}_{0.13}, \mathrm{Fe}_{0.91} \mathrm{Se}_{0.77} \mathrm{Te}_{0.23}$ films are about $4.46 \times 10^{5} \mathrm{~A} / \mathrm{cm}^{2}, 4.51 \times 10^{6} \mathrm{~A} / \mathrm{cm}^{2}$ and $4.05 \times 10^{6} \mathrm{~A} / \mathrm{cm}^{2}$, respectively. Compared with the three samples, it also confirms that the optimal composition is beneficial for $\mathrm{Fe}_{y} \mathrm{Se}_{1-x} \mathrm{Te}_{x}$ films exhibiting excellent superconductivity.

The interface structure plays a vital role in determining the superconducting properties for $\mathrm{Fe}_{y} \mathrm{Se}_{1-x} \mathrm{Te}_{x}$ films. Using the STEM analysis, we could reveal the 
$\mathrm{Fe}_{0.76} \mathrm{Se}_{0.87} \mathrm{Te}_{0.13} / \mathrm{TiO}_{2} / \mathrm{STO}$ microstructure and determined the morphology of the interface. The thicknesses of $\mathrm{Fe}_{0.76} \mathrm{Se}_{0.87} \mathrm{Te}_{0.13}$ and $\mathrm{TiO}_{2}$ film are about $32.4 \mathrm{~nm}$ and 29.5 nm, respectively. Figure 8 (a) shows the overview image of the $\mathrm{Fe}_{0.76} \mathrm{Se}_{0.87} \mathrm{Te}_{0.13} / \mathrm{TiO}_{2} / \mathrm{STO}$ interface. It can be seen that the heterostructure interface is sharp and clean without large-scale defects. The $\mathrm{TiO}_{2}$ buffer was successfully deposited between the $\mathrm{Fe}_{0.76} \mathrm{Se}_{0.87} \mathrm{Te}_{0.13}$ film and STO substrate. Figure 8 (b) shows the highmagnification HAADF image of $\mathrm{Fe}_{0.76} \mathrm{Se}_{0.87} \mathrm{Te}_{0.13} / \mathrm{TiO}_{2}$. The Fe, Se/Te, Ti and $\mathrm{O}$ atoms are arranged neatly at the interface. In this case, the $\mathrm{Fe}_{0.76} \mathrm{Se}_{0.87} \mathrm{Te}_{0.13}$ structure with a tetragonal space group $\mathrm{P} 4 / \mathrm{nmm}$ is very simple, and each unit cell contains 3 quintuple layers (QLs), which are bonded by van der Waals (vdW) [9]. The $\mathrm{TiO}_{2}$ unit cell has two Ti-O triple layers, which grow on STO along the $(00 l)$ direction. From Fig. 8 (b), a nanoscale damaged layer (or transition layer) was formed between the $\mathrm{TiO}_{2}$ and $\mathrm{Fe}_{0.76} \mathrm{Se}_{0.87} \mathrm{Te}_{0.13}$ interface. To determine the formation of this transition layer, the Atomic resolution EDX mapping was conducted in this area. The chemical elemental maps of Fig. 8 (c) confirm the suggestion from HAADF imaging that the atoms are arranged regularly without obvious diffusion and migration. Such high quality heterostructure had a significant influence on the enhancement of superconductivity for $\mathrm{Fe}_{y} \mathrm{Se}_{1-x} \mathrm{Te}_{x}$ films.

\section{Conclusion}

In summary, we successfully prepared the $\mathrm{Fe}_{y} \mathrm{Se}_{1-x} \mathrm{Te}_{x}$ thin films with $0.03 \leq x \leq$ 0.56 and $0.63 \leq y \leq 1.43$ by PLD. Our experimental results confirmed the significant deviation between the nominal compositions of targets and the real compositions of $\mathrm{Fe}_{y} \mathrm{Se}_{1-x} \mathrm{Te}_{x}$ films. Chemical composition does affect the superconducting properties such as $J_{c}$ and $H_{c 2}$ in $\mathrm{Fe}_{y} \mathrm{Se}_{1-x} \mathrm{Te}_{x}$ films. A new 3D phase diagram is presented from the experimental results of electrical transport, which reveals that the optimal composition for $\mathrm{Fe}_{y} \mathrm{Se}_{1-x} \mathrm{Te}_{x}$ films is $x=0.13-0.15$ and $y=0.73-0.78$. The field dependence of flux pinning energy displayed that the increase of Te doping can enhance the flux pinning in the $\mathrm{Fe}_{y} \mathrm{Se}_{1-x} \mathrm{Te}_{x}$ films. STEM investigation shows that the $\mathrm{Fe}_{0.76} \mathrm{Se}_{0.87} \mathrm{Te}_{0.13} / \mathrm{TiO}_{2} / \mathrm{STO}$ heterostructure has a sharp interface and exhibits almost no atomics intermixing. Our study results provide some further understanding on the 
mechanism of superconducting properties for $\mathrm{Fe}_{y} \mathrm{Se}_{1-x} \mathrm{Te}_{x}$ films, which has a certain guiding significance and reference value for the potential application of iron-based superconductors.

\section{Methods}

The PLD targets were prepared by the self-flux method with high purity materials (Fe 99.99\%, Te 99.999\% and Se 99.999\%) in the stoichiometric proportion. Fe, Se and Te were fully ground and squeezed into a $3 / 4$ inch block, and then encapsulated in a vacuum quartz tube. The vacuum quartz tube was calcined in a muffle furnace at $850{ }^{\circ} \mathrm{C}$ for 72 hours, then slowly cooled down to room temperature with the rate of $3{ }^{\circ} \mathrm{C} / \mathrm{min}$. The $\mathrm{Fe}_{y} \mathrm{Se}_{1-x} \mathrm{Te}_{x}$ epitaxial films were deposited on STO single crystalline substrates at $300{ }^{\circ} \mathrm{C}$ by PLD in a high vacuum $\left(\sim 10^{-7} \mathrm{mbar}\right)$. The distance between target and substrate was set at $\sim 70 \mathrm{~mm}$. A KrF excimer laser (248nm) was used for deposition with an energy density of $2.0 \mathrm{~J} / \mathrm{cm}^{2}$ and a repetition frequency of $2 \mathrm{~Hz}$. The size of the STO substrate is $5 \mathrm{~mm} \times 5 \mathrm{~mm}$. $\mathrm{TiO}_{2}$ film as a buffer layer was firstly deposited on STO substrate by PLD to improve the lattice matching between $\mathrm{Fe}_{y} \mathrm{Se}_{1-x} \mathrm{Te}_{x}$ film and STO substrate. The deposition temperature and deposition time for $\mathrm{Fe}_{y} \mathrm{Se}_{1-x} \mathrm{Te}_{x}$ and $\mathrm{TiO}_{2}$ film were $300{ }^{\circ} \mathrm{C}$ and $15 \mathrm{~min}, 600^{\circ} \mathrm{C}$ and $4.5 \mathrm{~min}$, respectively. After deposition, the films were annealed to room temperature at the rate of $5{ }^{\circ} \mathrm{C} / \mathrm{min}$.

X-ray diffraction (XRD) patterns using the $\theta / 2 \theta$ method were measured by Bruker D8 with $\mathrm{CuK} \alpha$ radiation $\left(\lambda=1.54 \AA\right.$ ). The chemical composition of $\mathrm{Fe}_{y} \mathrm{Se}_{1-x} \mathrm{Te}_{x}$ films was determined by energy dispersive $x$-ray spectroscopy (EDX) in a Gemini 500 scanning electron microscope (SEM) mapping. The measurements of electrical transport were carried out via the physical property measurement system (PPMS-9 T, Quantum Design). Magnetization measurements on films with 100 Oe/s of sweep rate were performed in vibrating sample magnetometer (VSM). The microstructures of $\mathrm{Fe}_{y} \mathrm{Se}_{1-x} \mathrm{Te}_{x}$ films were examined by scanning transmission electron microscopy (STEM, FEI Titan G2 60-300 aberration). Samples for the STEM were cut and milled in a focused ion beam (FIB, FEI Helios Nanolab 600) according to the so-called microbridge sampling technique. 



\section{Acknowledgements}

The authors are grateful for the financial support of the Ministry of Science and Technology of China (2017YFA0303200), the National Natural Science Foundation of China (11671203), and the National Key Research and Development Program of China (2016YFA0300401).

\section{Author Contributions}

Yalin Zhang, Tong Wang, Zhihe Wang and Zhongwen Xing conceived the experiments; Yalin Zhang conducted the experiments; Yalin Zhang, Zhihe Wang, and Zhongwen Xing analysed the results; Yalin Zhang and Zhihe Wang wrote the paper. All authors have reviewed the manuscript.

\section{Declaration of competing interest}

The authors declare no competing financial interests. 


\section{Reference}

[1] Kamihara, Y. et al. Iron-based layered superconductor $\mathrm{La}\left[\mathrm{O}_{1-x} \mathrm{~F}_{x}\right]$ FeAs $(x=0.05-0.12)$ with $T_{c}$ $=26$ K. J. Am. Chem. Soc. 130, 3296 (2008).

[2] Hsu, F. C. et al. Superconductivity in the PbO-type structure $\alpha$-FeSe. P. Natl. Acad. Sci. USA 105, 14262 (2008).

[3] Medvedev, S. et al. Electronic and magnetic phase diagram of $\beta-\mathrm{Fe}_{1.01} \mathrm{Se}$ with superconductivity at $36.7 \mathrm{~K}$ under pressure. Nat. Mater. 8, 630 (2009).

[4] Margadonna, S. et al. Pressure evolution of the low-temperature crystal structure and bonding of the superconductor FeSe $\left(T_{c}=37\right.$ K). Phys. Rev. B 80, 064506 (2009).

[5] Ge, J. F. et al. Superconductivity above $100 \mathrm{~K}$ in single-layer FeSe films on doped $\mathrm{SrTiO}_{3}$. Nat. Mater. 14, 285 (2015).

[6] Hanzawa, K. et al. Electric field-induced superconducting transition of insulating FeSe thin film at 35 K. Proc. Natl. Acad. Sci. USA 113, 3986 (2016).

[7] Zhang, H. et al. Origin of charge transfer and enhanced electron-phonon coupling in single unitcell FeSe films on $\mathrm{SrTiO}_{3}$. Nat. Commun. 8, 1 (2017).

[8] Zhou, G. Y. et al. Interface enhanced superconductivity in monolayer FeSe films on MgO (001): charge transfer with atomic substitution. Sci. Bull. 63, 747 (2018).

[9] Sims, H. et al. Intrinsic interfacial van der Waals monolayers and their effect on the hightemperature superconductor $\mathrm{FeSe} / \mathrm{SrTiO}_{3}$. Phys. Rev. B 100, 144103 (2019).

[10] Li, F. S. et al. Atomically resolved $\mathrm{FeSe} / \mathrm{SrTiO}_{3}$ (001) interface structure by scanning transmission electron microscopy. 2D Mater. 3, 024002 (2016).

[11] Yeh, K. W. et al. Tellurium substitution effect on superconductivity of the $\alpha$-phase iron selenide. Europhys Lett. 84, 37002 (2008).

[12] Fang, M. H. et al. Superconductivity close to magnetic instability in $\mathrm{Fe}\left(\mathrm{Se}_{1-x} \mathrm{Te}_{x}\right)_{0.82}$. Phys. Rev. $B$ 78, 224503 (2008).

[13] Liu, T. J. et al. From $(\pi, 0)$ magnetic order to superconductivity with $(\pi, \pi)$ magnetic resonance in $\mathrm{Fe}_{1.02} \mathrm{Te}_{1-x} \mathrm{Se}_{x}$. Nat. Mater. 9, 718 (2010).

[14] Imai, Y. et al. Suppression of phase separation and giant enhancement of superconducting transition temperature in $\mathrm{FeSe}_{1-x} \mathrm{Te}_{x}$ thin films. Proc. Natl. Acad. Sci. USA 112, 1937 (2015). 
[15] Bellingeri, E. et al. $T_{c}=21 \mathrm{~K}$ in epitaxial $\mathrm{FeSe}_{0.5} \mathrm{Te}_{0.5}$ thin films with biaxial compressive strain. Appl. Phys. Lett. 96, 102512 (2010).

[16] Huang, S. X. et al. Control of tetrahedral coordination and superconductivity in $\mathrm{FeSe}_{0.5} \mathrm{Te}_{0.5}$ thin films. Phys. Rev. Lett. 104, 217002 (2010).

[17] Iida, K. et al. Generic Fe buffer layers for Fe-based superconductors: Epitaxial $\mathrm{FeSe}_{1-x} \mathrm{Te}_{x}$ thin films. Appl. Phys. Lett. 99, 202503 (2011).

[18] Bellingeri, E. et al. Tuning of the superconducting properties of $\mathrm{FeSe}_{0.5} \mathrm{Te}_{0.5}$ thin films through the substrate effect. Supercond. Sci. Technol. 25, 084022 (2012).

[19] Iida, K. et al. Intrinsic pinning and the critical current scaling of clean epitaxial Fe (Se, Te) thin films. Phys. Rev. B 87, 104510 (2013).

[20] Ichinose, A. et al. Microscopic analysis of the chemical reaction between $\mathrm{Fe}(\mathrm{Te}, \mathrm{Se})$ thin films and underlying $\mathrm{CaF}_{2}$. Supercond. Sci. Technol. 26, 075002 (2013).

[21] Zhuang, J. C. et al. Pauli-limited effect in the magnetic phase diagram of $\mathrm{FeSe}_{x} \mathrm{Te}_{1-x}$ thin films. Appl. Phys. Lett. 107, 222601 (2015).

[22] Zhuang, J. C. et al. Enhancement of transition temperature in $\mathrm{Fe}_{x} \mathrm{Se}_{0.5} \mathrm{Te}_{0.5}$ film via iron vacancies. Appl. Phys. Lett. 104, 262601 (2014).

[23] Huang, J. F. et al. Linear correlation between the c-axis lattice constant and superconducting critical temperature in $\mathrm{FeSe}_{0.5} \mathrm{Te}_{0.5}$ thin films. Mater. Res. Express 7, 046002 (2020).

[24] Wu, Z. F. et al. Anisotropic flux pinning energy in $\mathrm{FeSe}_{x} \mathrm{Te}_{1-x}$ single crystals. Physica. $C \mathbf{5 2 8}$, 39 (2016).

[25] Zhuang, J. C. et al. Pauli-limited effect in the magnetic phase diagram of $\mathrm{FeSe}_{x} \mathrm{Te}_{1-x}$ thin films. Sci. Rep. 4, 1 (2014).

[26] Imai, Y. et al. Control of structural transition in $\mathrm{FeSe}_{1-x} \mathrm{Te}_{x}$ thin films by changing substrate materials. Sci. Rep. 7, 1 (2017).

[27] Chen, T. K. et al. Fe-vacancy order and superconductivity in tetragonal $\beta$-Fe $\mathrm{Fe}_{1-x} \mathrm{Se}$. Proc. Natl. Acad. Sci. USA 111, 63 (2014).

[28] Bendele, M. et al. Tuning the superconducting and magnetic properties of $\mathrm{Fe}_{y} \mathrm{Se}_{0.25} \mathrm{Te}_{0.75}$ by varying the iron content. Phys. Rev. B 82, 212504 (2010).

[29] Werthamer, N. R. et al. Temperature and purity dependence of the superconducting critical field, $\mathrm{H}_{\mathrm{c} 2}$. III. Electron spin and spin-orbit effects. Phys. Rev. 147, 295 (1966). 
[30] Palstra, T. T. M. et al. Thermally activated dissipation in $\mathrm{Bi}_{2.2} \mathrm{Sr}_{2} \mathrm{Ca}_{0.8} \mathrm{Cu}_{2} \mathrm{O}_{8+\delta}$. Phys. Rev. Lett. 61, 1662 (1988).

[31] Palstra, T. T. M. et al. Dissipative flux motion in high-temperature superconductors. Phys. Rev. B 41, 6621 (1990).

[32] Xing, X. Z. et al. Thermally activated flux flow, vortex-glass phase transition and the mixedstate Hall effect in 112-type iron pnictide superconductors. Sci. China Phys. Mec. 61, 127406 (2018) [33] Kucera, J. T. et al. Magnetic-field and temperature dependence of the thermally activated dissipation in thin films of $\mathrm{Bi}_{2} \mathrm{Sr}_{2} \mathrm{CaCu}_{2} \mathrm{O}_{8+\delta}$. Phys. Rev. B 46, 11004 (1992).

[34] Mao, R. et al. Effect of Te-doping on the superconducting characteristics of FeSe single crystal. J. Alloy. Compd. 809, 151851 (2019).

[35] Bean, C. P. Magnetization of high-field superconductors. Rev. Mod. Phys. 36, 31 (1964). 


\section{Figure captions}

Fig. 1. X-ray diffraction patterns of $\mathrm{Fe}_{y} \mathrm{Se}_{1-x} \mathrm{Te}_{x}$ thin films.

Fig. 2. (a), (b) Temperature dependence of resistivity from 2 to $300 \mathrm{~K}$ for $\mathrm{Fe}_{y} \mathrm{Se}_{1-x} \mathrm{Te}_{x}$ thin films. (a) $(x, y)=(0.03,0.63),(0.06,0.72),(0.13,0.76),(0.23,0.91),(0.34,1.09)$ and $(0.56,1.43)$. Inset: enlarged plot for the definition of normal-state resistivity $\rho_{\mathrm{n}}$. (b) $(x, y)=(0.15,0.73),(0.13,0.76)$ and $(0.16,0.78)$. Inset: the enlarged $\rho-T$ curve near $T_{c}$. (c) Sketch of the proposed temperature doping 3D phase diagram for $\mathrm{Fe}_{y} \mathrm{Se}_{1-x} \mathrm{Te}_{x}$ superconducting system, showing regions of superconductivity (SC), flux flow (FF) and normal state (NS).

Fig. 3. Temperature dependence of resistivity near $T_{c}$ in various magnetic fields for $\mathrm{Fe}_{0.76} \mathrm{Se}_{0.87} \mathrm{Te}_{0.13}$ thin film. (a) $H / / c$-axis and (b) $H / / a b$-axis.

Fig. 4. Upper critical field versus temperature phase diagram determined by $\rho / \rho_{n}=90 \%$. (a) $\mathrm{Fe}_{0.72} \mathrm{Se}_{0.94} \mathrm{Te}_{0.06}$, (b) $\mathrm{Fe}_{0.76} \mathrm{Se}_{0.87} \mathrm{Te}_{0.13}$, (c) $\mathrm{Fe}_{0.91} \mathrm{Se}_{0.77} \mathrm{Te}_{0.23}$.

Fig. 5. $\ln \rho$ versus $1 / \mathrm{T}$ curves in various magnetic fields of $\mathrm{Fe}_{0.76} \mathrm{Se}_{0.87} \mathrm{Te}_{0.13}$ thin film. (a) $H / / c$-axis; (b) $H / / a b$-axis. (c) Magnetic field dependence of the effective flux pinning energy for $\mathrm{Fe}_{0.72} \mathrm{Se}_{0.94} \mathrm{Te}_{0.06}, \mathrm{Fe}_{0.76} \mathrm{Se}_{0.87} \mathrm{Te}_{0.13}$ and $\mathrm{Fe}_{0.91} \mathrm{Se}_{0.77} \mathrm{Te}_{0.23}$ thin films.

Fig. 6. Magnetic hysteresis loops of $\mathrm{Fe}_{0.91} \mathrm{Se}_{0.77} \mathrm{Te}_{0.23}$ film at various temperatures in magnetic field parallel to the $c$-axis.

Fig. 7. Magnetic field dependence of critical current density $J_{c}$ at various temperatures for $\mathrm{Fe}_{0.72} \mathrm{Se}_{0.94} \mathrm{Te}_{0.06}, \mathrm{Fe}_{0.76} \mathrm{Se}_{0.87} \mathrm{Te}_{0.13}$ and $\mathrm{Fe}_{0.91} \mathrm{Se}_{0.77} \mathrm{Te}_{0.23}$ thin films.

Fig. 8. (a) Overview image of the $\mathrm{Fe}_{0.76} \mathrm{Se}_{0.87} \mathrm{Te}_{0.13} / \mathrm{TiO}_{2} / \mathrm{STO}$ thin film interface. (b) Atomically resolved HADDF-STEM image of $\mathrm{Fe}_{0.76} \mathrm{Se}_{0.87} \mathrm{Te}_{0.13} / \mathrm{TiO}_{2}$ heterostructure. (c) EDX-mapping results shows the distribution of $\mathrm{Fe}$ (red), Se (green), Te (blue) $\mathrm{Ti}$ (magenta), O (cyan).

\section{Table captions}

Table 1. The composition, onset and zero-resistivity temperature, and $c$-axis lattice parameter of thin films for nominal composition $\mathrm{FeSe}_{1-x} \mathrm{Te}_{x}$ targets.

Table 2. The composition, onset and zero-resistivity temperature, and $c$-axis lattice parameter of thin films for nominal composition $\mathrm{Fe}_{y} \mathrm{Se}_{0.4} \mathrm{Te}_{0.6}$ targets. 
Figure 1

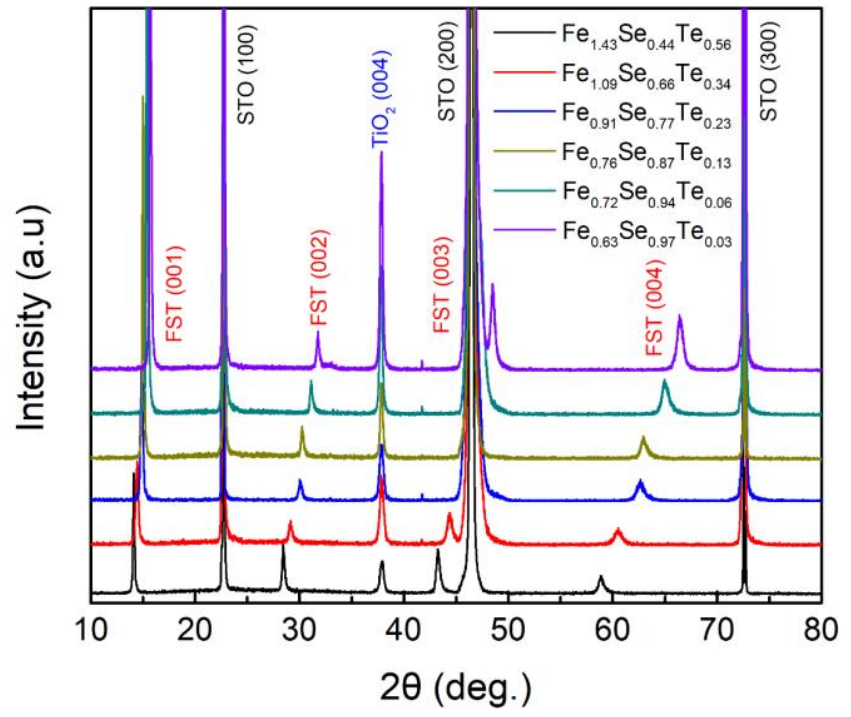


Figure 2
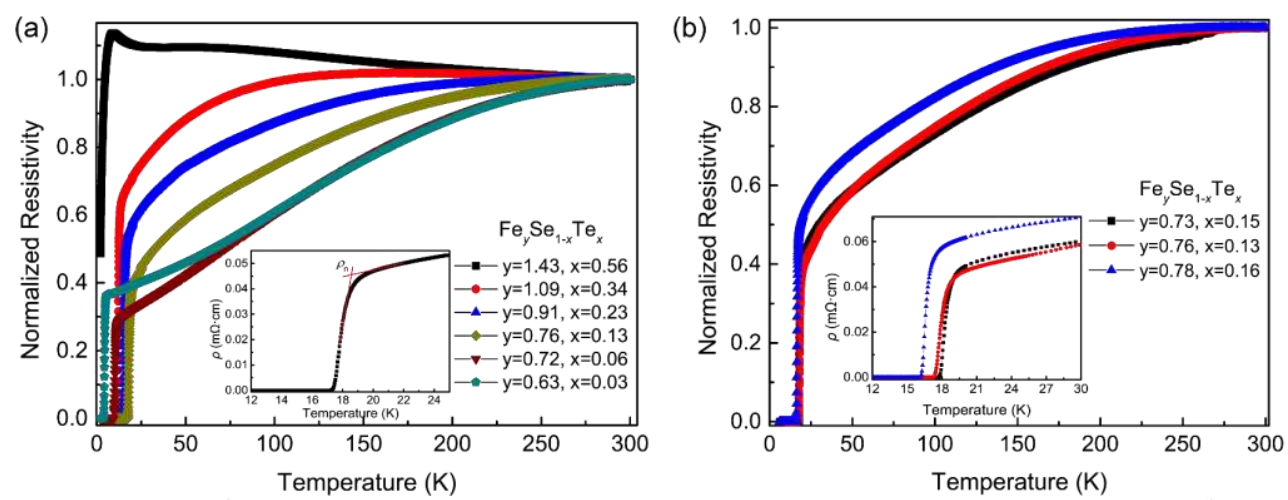

(c)

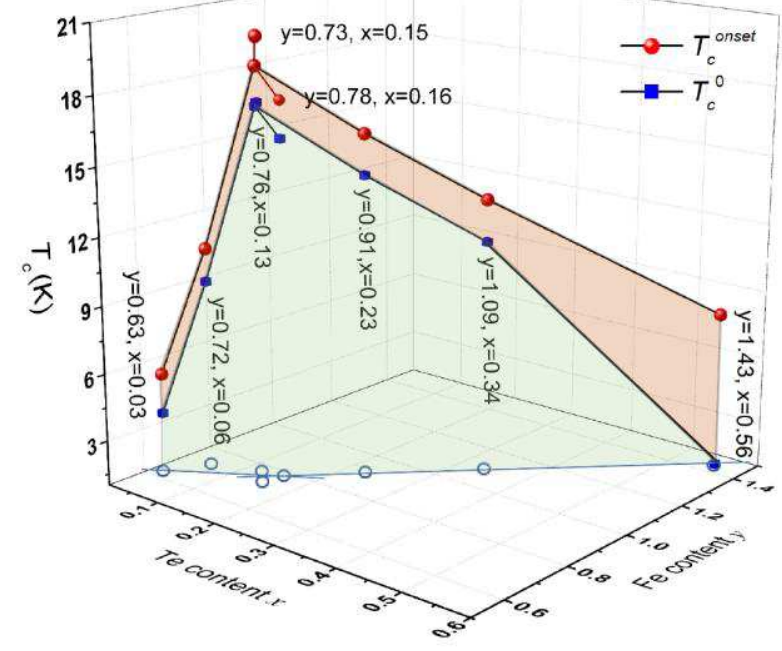


Figure 3

(a)

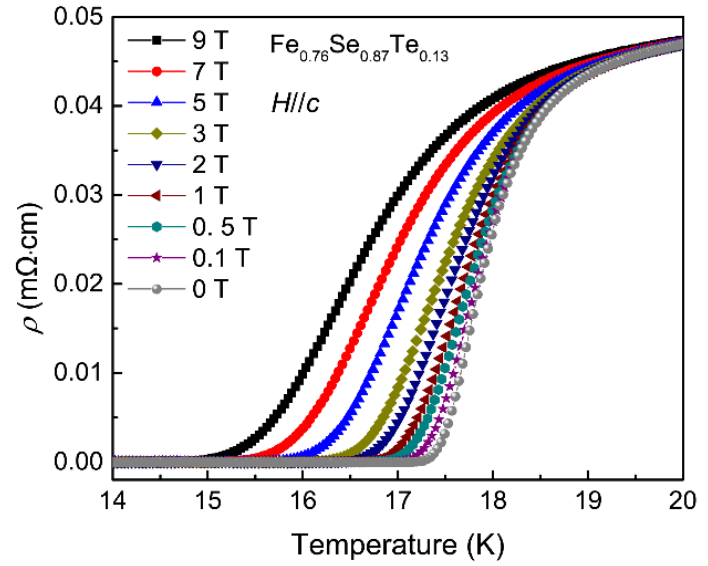

(b)

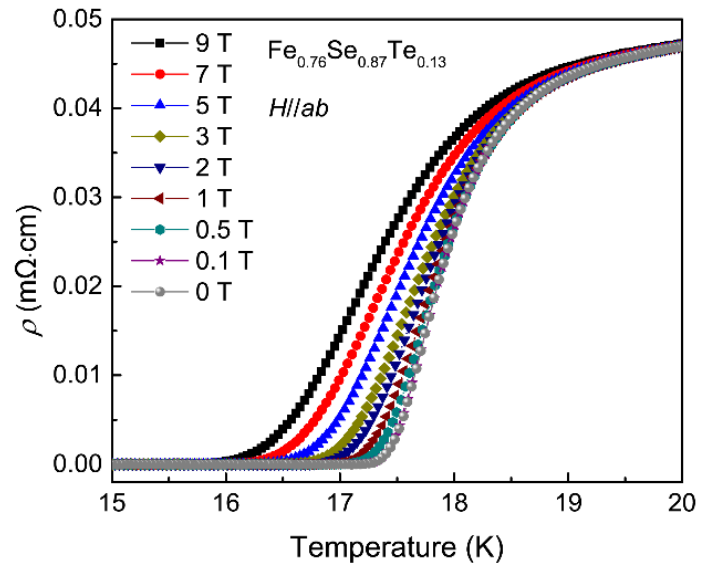


Figure 4

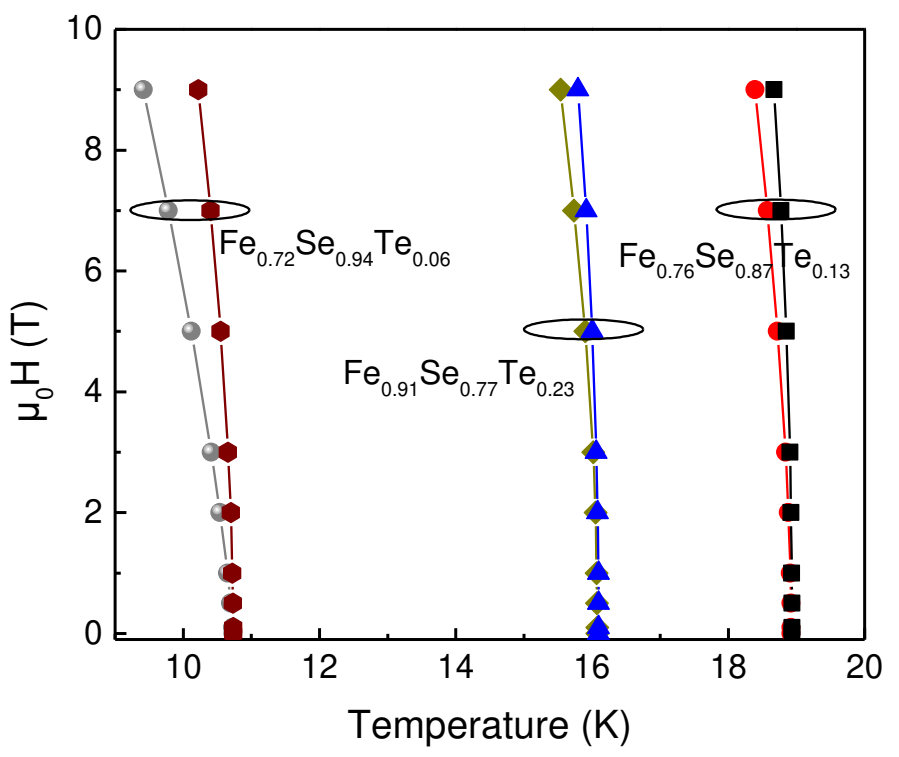




\section{Figure 5}

(a)

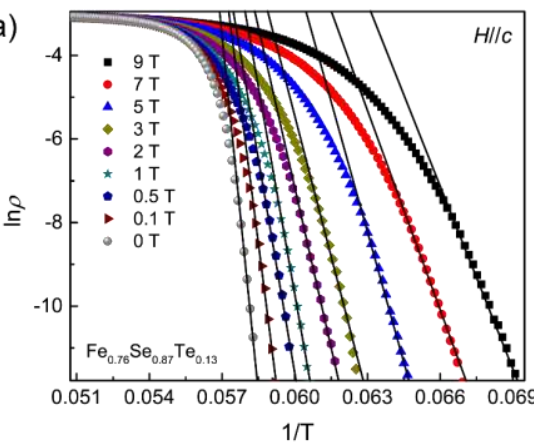

(b)

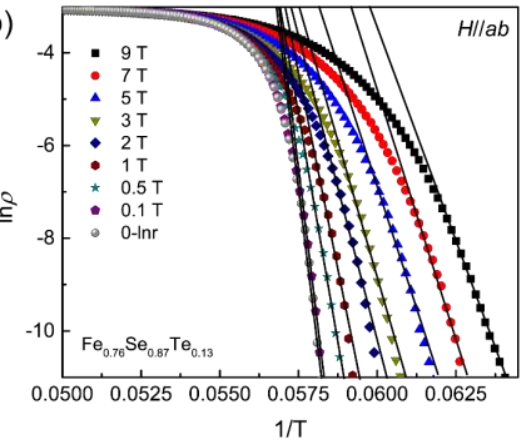

(c)

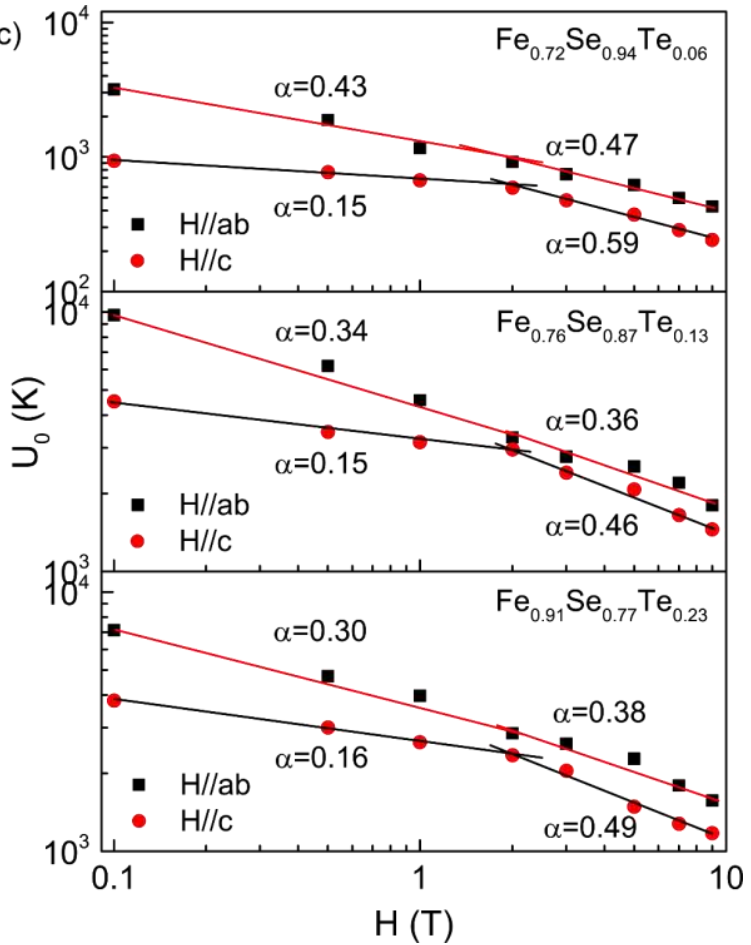


Figure 6

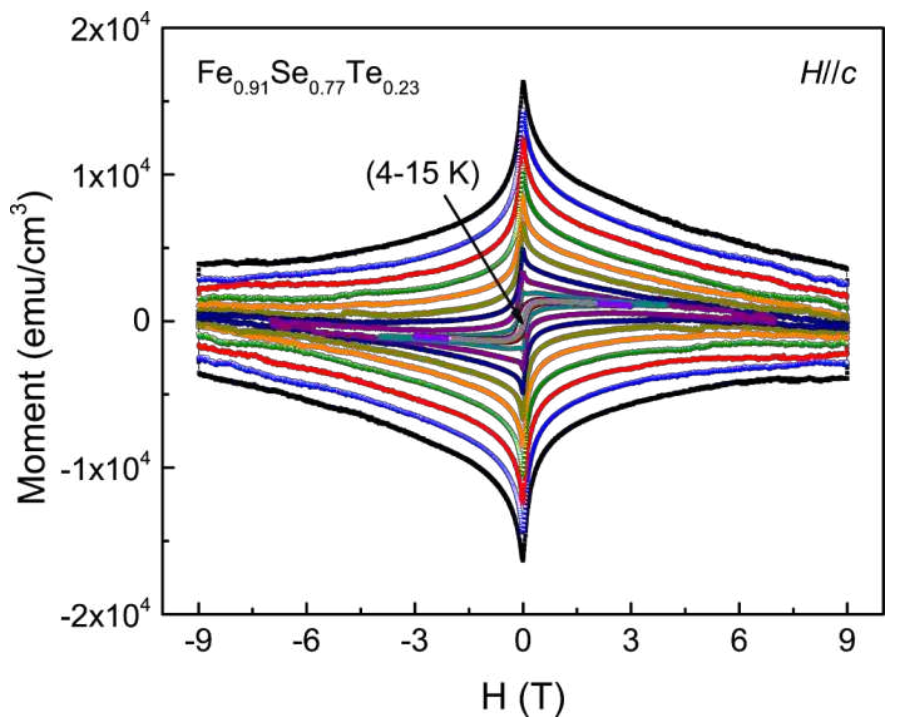


Figure 7

(a)
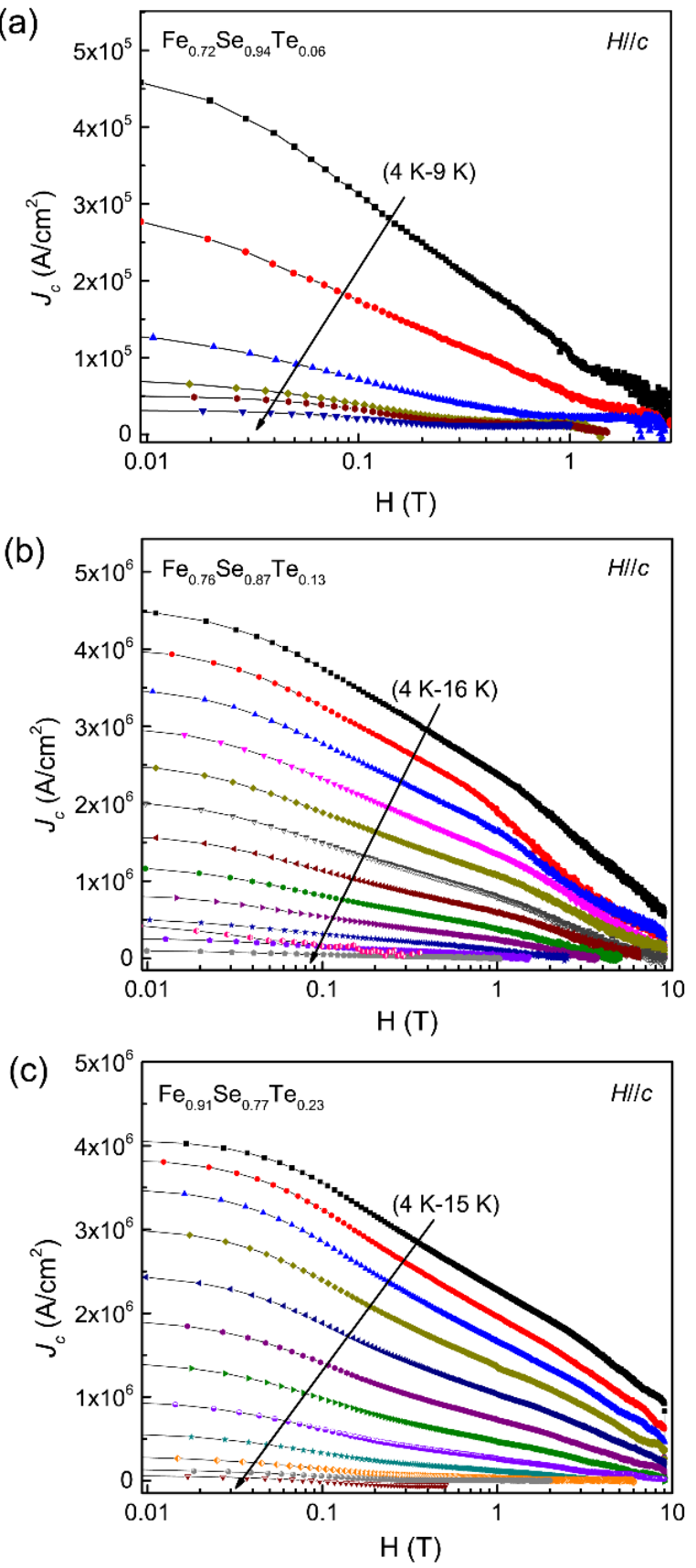
Figure 8

(a)

(b)

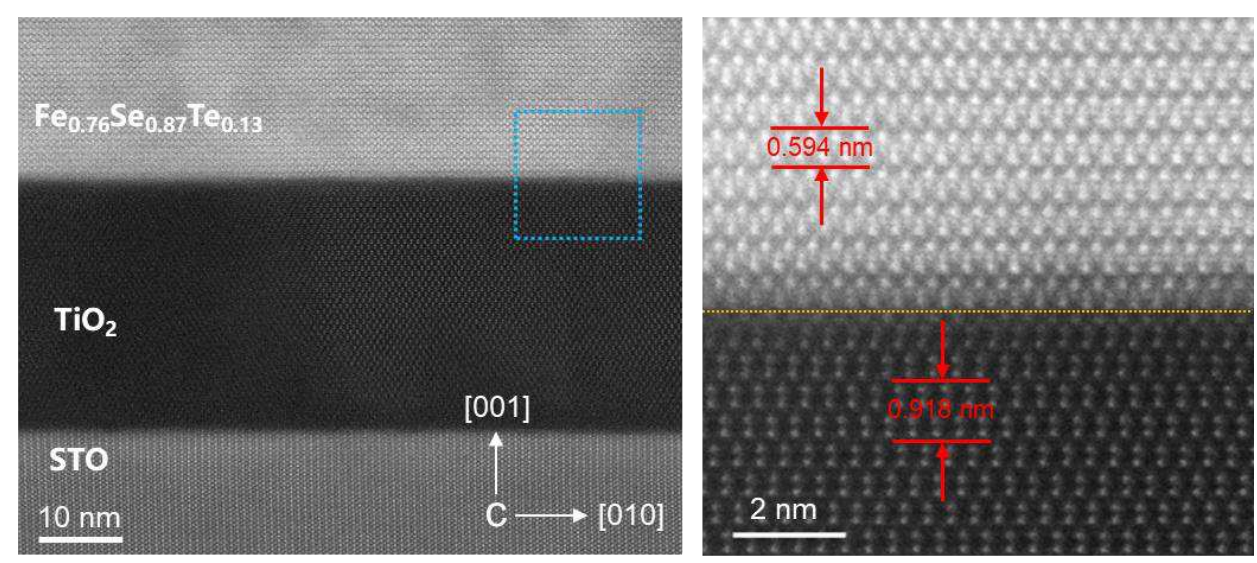

(c)

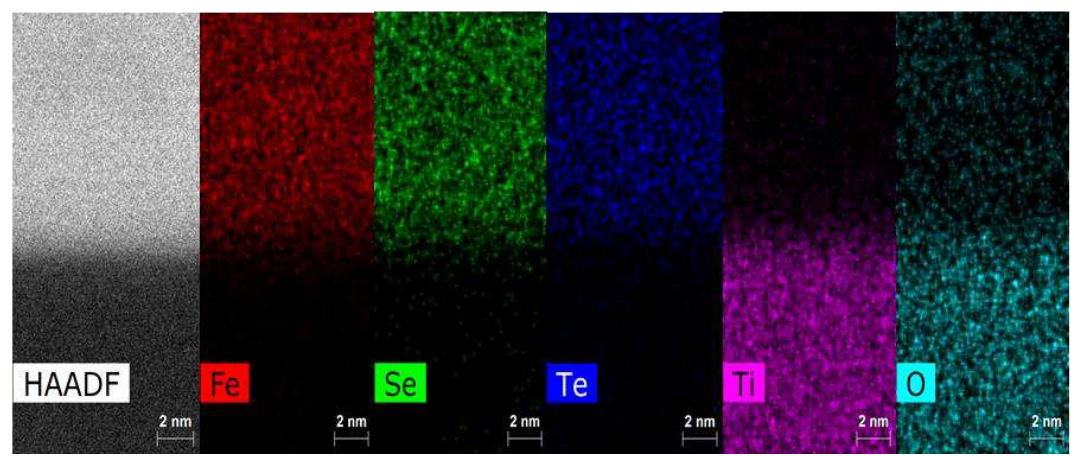


Table 1

\begin{tabular}{ccccc}
\hline nominal composition & real composition $( \pm 0.02)$ & $T_{c}^{\text {onset }}(\mathrm{K})$ & $T_{c}^{0}(\mathrm{~K})$ & $c$ parameter $(\AA)$ \\
\hline $\mathrm{FeSe}_{0.6} \mathrm{Te}_{0.4}$ & $\mathrm{Fe}_{0.63} \mathrm{Se}_{0.97} \mathrm{Te}_{0.03}$ & 5.49 & 3.71 & 5.6361 \\
$\mathrm{FeSe}_{0.5} \mathrm{Te}_{0.5}$ & $\mathrm{Fe}_{0.72} \mathrm{Se}_{0.94} \mathrm{Te}_{0.06}$ & 10.73 & 9.44 & 5.7526 \\
$\mathrm{FeSe}_{0.4} \mathrm{Te}_{0.6}$ & $\mathrm{Fe}_{0.76} \mathrm{Se}_{0.87} \mathrm{Te}_{0.13}$ & 18.95 & 17.34 & 5.8398 \\
$\mathrm{FeSe}_{0.3} \mathrm{Te}_{0.7}$ & $\mathrm{Fe}_{0.91} \mathrm{Se}_{0.77} \mathrm{Te}_{0.23}$ & 16.13 & 14.35 & 5.9486 \\
$\mathrm{FeSe}_{0.2} \mathrm{Te}_{0.8}$ & $\mathrm{Fe}_{1.09} \mathrm{Se}_{0.66} \mathrm{Te}_{0.34}$ & 13.21 & 11.37 & 6.0502 \\
$\mathrm{FeSe}_{0.1} \mathrm{Te}_{0.9}$ & $\mathrm{Fe}_{1.43} \mathrm{Se}_{0.44} \mathrm{Te}_{0.56}$ & 8.03 & - & 6.1973 \\
\hline
\end{tabular}

Table 2

\begin{tabular}{ccccc}
\hline nominal composition & real composition $( \pm 0.02)$ & $T_{c}{ }^{\text {onset }}(\mathrm{K})$ & $T_{c}{ }^{0}(\mathrm{~K})$ & $c$ parameter $(\AA)$ \\
\hline $\mathrm{Fe}_{0.9} \mathrm{Se}_{0.4} \mathrm{Te}_{0.6}$ & $\mathrm{Fe}_{0.73} \mathrm{Se}_{0.85} \mathrm{Te}_{0.15}$ & 20.35 & 17.55 & 5.7287 \\
$\mathrm{FeSe}_{0.4} \mathrm{Te}_{0.6}$ & $\mathrm{Fe}_{0.76} \mathrm{Se}_{0.87} \mathrm{Te}_{0.13}$ & 18.95 & 17.34 & 5.8398 \\
$\mathrm{Fe}_{1.1} \mathrm{Se}_{0.4} \mathrm{Te}_{0.6}$ & $\mathrm{Fe}_{0.78} \mathrm{Se}_{0.84} \mathrm{Te}_{0.16}$ & 17.64 & 16.01 & 6.0047 \\
\hline
\end{tabular}

